DRAFT VERSION JANUARY 10, 2012

Preprint typeset using $\mathrm{LATE}_{\mathrm{E}} \mathrm{X}$ style emulateapj v. 5/2/11

\title{
PG 1553+113: FIVE YEARS OF OBSERVATIONS WITH MAGIC
}

J. Aleksić ${ }^{1}$, E. A. Alvarez ${ }^{2}$, L. A. Antonelli ${ }^{3}$, P. Antoranz ${ }^{4}$, M. Asensio ${ }^{2}$, M. Backes $^{5}$, J. A. Barrio $^{2}$, D. Bastieri ${ }^{6}$, J. Becerra GonzÁlez ${ }^{7,8}$, W. Bednarek ${ }^{9}$, A. Berdyugin ${ }^{10}$, K. Berger ${ }^{7,8}$, E. Bernardini ${ }^{11}$, A. Biland ${ }^{12}$, O. Blanch ${ }^{1}$, R. K. Bock ${ }^{13}$, A. Boller ${ }^{12}$, G. Bonnoli ${ }^{3}$, D. Borla Tridon ${ }^{13}$, I. Braun ${ }^{12}$, T. Bretz ${ }^{14,26}$, A. Cañellas ${ }^{15}$, E. Carmona ${ }^{13}$, A. Carosi ${ }^{3}$, P. Colin $^{13}$, E. Colombo ${ }^{7}$, J. L. Contreras ${ }^{2}$, J. Cortina ${ }^{1}$, L. Cossio ${ }^{16}$, S. Covino ${ }^{3}$, F. Dazzi ${ }^{16,27}$, A. De Angelis ${ }^{16}$, G. De Caneva ${ }^{11}$, E. De Cea del Pozo ${ }^{17}$, B. De Lotto ${ }^{16}$, C. Delgado Mendez $^{7,28}$, A. Diago Ortega ${ }^{7,8}$, M. Doert $^{5}$, A. Domínguez ${ }^{18}$, D. Dominis Prester ${ }^{19}$, D. Dorner $^{12}$, M. Doro $^{20}{ }$ D. Elsaesser ${ }^{14}$, D. Ferenc ${ }^{19}$, M. V. Fonseca ${ }^{2}$, L. Font ${ }^{20}$, C. Fruck ${ }^{13}$, R. J. García López ${ }^{7,8}$, M. GarczarczyK ${ }^{7}$, D. Garrido ${ }^{20}$, G. Giavitto ${ }^{1}$, N. Godinović ${ }^{19}$, D. Hadasch ${ }^{17}$, D. HäFner ${ }^{13}$, A. Herrero ${ }^{7,8}$, D. Hildebrand ${ }^{12}$, D. Höhne-Mönch ${ }^{14}$, J. Hose ${ }^{13}$, D. Hrupec ${ }^{19}$, B. Huber ${ }^{12}$, T. Jogler ${ }^{13}$, H. Kellermann ${ }^{13}$, S. KlePser ${ }^{1}$, T. Krähenbühl ${ }^{12}$, J. Krause ${ }^{13}$, A. La Barbera ${ }^{3}$, D. Lelas ${ }^{19}$, E. LeOnardo ${ }^{4}$, E. Lindfors ${ }^{10}$, S. LOMbardi $^{6}$, M. López ${ }^{2}$, A. López-Oramas ${ }^{1}$, E. Lorenz ${ }^{12,13}$, M. Makariev ${ }^{21}$, G. Maneva ${ }^{21}$, N. Mankuzhiyil ${ }^{16}$, K. Mannheim ${ }^{14}$, L. Maraschi ${ }^{3}$, M. Mariotti ${ }^{6}$, M. Martínez ${ }^{1}$, D. Mazin ${ }^{1,13}$, M. MeucCi $^{4}$, J. M. Miranda ${ }^{4}$, R. Mirzoyan ${ }^{13}$, H. Miyamoto ${ }^{13}$, J. Moldón ${ }^{15}$, A. Moralejo ${ }^{1}$, P. Munar-Adrover ${ }^{15}$, D. Nieto ${ }^{2}$, K. Nilsson ${ }^{10,29}$, R. Orito $^{13}$, I. Oya ${ }^{2}$, D. Paneque ${ }^{13}$, R. PAoletti ${ }^{4}$, S. Pardo ${ }^{2}$, J. M. PARedes ${ }^{15}$, S. PARtini ${ }^{4}$, M. Pasanen ${ }^{10}$, F. Pauss ${ }^{12}$, M. A. Perez-Torres ${ }^{1}$, M. Persic $^{16,22}$, L. Peruzzo ${ }^{6}$, M. Pilia ${ }^{23}$, J. Pochon ${ }^{7}$, F. Prada ${ }^{18}$, P. G. Prada Moroni ${ }^{24}$, E. Prandini ${ }^{6, *}$, I. Puljak ${ }^{19}$, I. Reichardt ${ }^{1}$, R. Reinthal ${ }^{10}$, W. Rhode ${ }^{5}$, M. Ribó ${ }^{15}$, J. Rico ${ }^{25,1}$, S. Rügamer $^{14}$, A. Saggion ${ }^{6}$, K. Saito ${ }^{13}$, T. Y. Saito ${ }^{13}$, M. Salvati ${ }^{3}$, K. Satalecka ${ }^{2}$, V. Scalzotto ${ }^{6}$, V. Scapin ${ }^{2}$, C. Schultz ${ }^{6}$, T. Schweizer ${ }^{13}$, M. Shayduk ${ }^{13}$, S. N. Shore ${ }^{24}$, A. Sillanpä̈̈ ${ }^{10}$, J. Sitarek ${ }^{9}$, D. Sobczynska ${ }^{9}$, F. Spanier ${ }^{14}$,

S. Spiro ${ }^{3}$, V. Stamatescu ${ }^{1}$, A. Stamerra ${ }^{4}$, B. Steinke ${ }^{13}$, J. Storz ${ }^{14}$, N. Strah ${ }^{5}$, T. Surićl9, L. Takalo ${ }^{10}$,

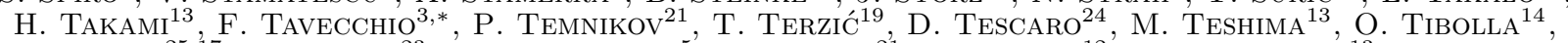
D. F. Torres ${ }^{25}, 17$, A. Treves ${ }^{23}$, M. Uellenbeck ${ }^{5}$, H. VAnkov $^{21}$, P. Vogler ${ }^{12}$, R. M. WAGneR ${ }^{13}$, Q. Weitzel ${ }^{12}$,

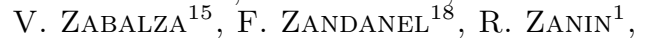

AND,

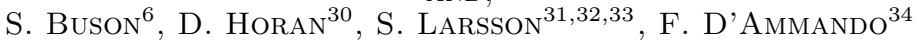

${ }^{1}$ IFAE, Edifici Cn., Campus UAB, E-08193 Bellaterra, Spain

2 Universidad Complutense, E-28040 Madrid, Spain

3 INAF National Institute for Astrophysics, I-00136 Rome, Italy

${ }^{4}$ Università di Siena, and INFN Pisa, I-53100 Siena, Italy

5 Technische Universität Dortmund, D-44221 Dortmund, Germany

${ }^{6}$ Università di Padova and INFN, I-35131 Padova, Italy

7 Inst. de Astrofísica de Canarias, E-38200 La Laguna, Tenerife, Spain

8 Depto. de Astrofísica, Universidad de La Laguna, E-38206 La Laguna, Spain

9 University of Łódź, PL-90236 Lodz, Poland

10 Tuorla Observatory, University of Turku, FI-21500 Piikkiö, Finland

11 Deutsches Elektronen-Synchrotron (DESY), D-15738 Zeuthen, Germany

12 ETH Zurich, CH-8093 Switzerland

13 Max-Planck-Institut für Physik, D-80805 München, Germany

${ }^{14}$ Universität Würzburg, D-97074 Würzburg, Germany

15 Universitat de Barcelona (ICC/IEEC), E-08028 Barcelona, Spain

16 Università di Udine, and INFN Trieste, I-33100 Udine, Italy

17 Institut de Ciències de l'Espai (IEEC-CSIC), E-08193 Bellaterra, Spain

18 Inst. de Astrofísica de Andalucía (CSIC), E-18080 Granada, Spain

19 Croatian MAGIC Consortium, Rudjer Boskovic Institute, University of Rijeka and University of Split, HR-10000 Zagreb, Croatia 20 Universitat Autònoma de Barcelona, E-08193 Bellaterra, Spain

21 Inst. for Nucl. Research and Nucl. Energy, BG-1784 Sofia, Bulgaria

${ }^{22}$ INAF/Osservatorio Astronomico and INFN, I-34143 Trieste, Italy

23 Università dell'Insubria, Como, I-22100 Como, Italy

${ }^{24}$ Università di Pisa, and INFN Pisa, I-56126 Pisa, Italy 25 ICREA, E-08010 Barcelona, Spain

26 now at: Ecole polytechnique fédérale de Lausanne (EPFL), Lausanne, Switzerland 27 supported by INFN Padova

28 now at: Centro de Investigaciones Energéticas, Medioambientales y Tecnológicas (CIEMAT), Madrid, Spain

29 now at: Finnish Centre for Astronomy with ESO (FINCA), University of Turku, Finland

${ }^{30}$ Laboratoire Leprince-Ringuet, Ecole polytechnique, CNRS/IN2P3, Palaiseau, France

31 Department of Physics, Stockholm University, AlbaNova, SE-106 91 Stockholm, Sweden

32 The Oskar Klein Center for Cosmoparticle Physics, AlbaNova, SE-106 91 Stockholm, Sweden

33 Department of Astronomy, Stockholm University, SE-106 91 Stockholm, Sweden

${ }^{34}$ Consorzio Interuniversitario Fisica Spaziale (CIFS), v.le Settimio Severo 63, I-10133 Torino, Italy and

* corresponding authors. E-mail: elisa.prandini@pd.infn.it, fabrizio.tavecchio@brera.inaf.it. Draft version January 10, 2012

\section{ABSTRACT}

We present the results of five years (2005-2009) of MAGIC observations of the BL Lac object PG 1553+113 at very high energies (VHEs, E > $100 \mathrm{GeV}$ ). Power law fits of the individual years are compatible with a steady mean photon index $\Gamma=4.27 \pm 0.14$. In the last three years of data, the 
flux level above $150 \mathrm{GeV}$ shows a clear variability (probability of constant flux $<0.001 \%$ ). The flux variations are modest, lying in the range from $4 \%$ to $11 \%$ of the Crab Nebula flux. Simultaneous optical data also show only modest variability that seems to be correlated with VHE gamma ray variability. We also performed a temporal analysis of (all available) simultaneous Fermi/LAT data of PG $1553+113$ above $1 \mathrm{GeV}$, which reveals hints of variability in the 2008-2009 sample. Finally, we present a combination of the mean spectrum measured at very high energies with archival data available for other wavelengths. The mean spectral energy distribution can be modeled with a onezone Synchrotron Self Compton (SSC) model, which gives the main physical parameters governing the VHE emission in the blazar jet.

Subject headings: radiation mechanisms: non-thermal, gamma-rays: observations, BL Lacertae objects: individual (PG 1553+113)

\section{INTRODUCTION}

The majority of extragalactic $\gamma$ ray sources, both at $\mathrm{GeV}$ energies and above $100 \mathrm{GeV}$, are blazars, radioloud active galactic nuclei with a relativistic jet pointing towards the Earth. Their emission is dominated by the non-thermal continuum produced within the jet and boosted by relativistic effects (Urry \& Padovani 1995). The spectral energy distribution (SED) displays two broad peaks, widely interpreted as due to synchrotron, low frequency peak, and inverse Compton, high frequency peak, mechanism (although the high energy peak could also be the result of hadronic processes, as proposed in Mannheim 1993). Among blazars, BL Lac objects are characterized by extremely weak emission lines in their optical spectra, which often makes a measurement of their redshift difficult. The large majority of extragalactic sources detected above $100 \mathrm{GeV}$ are BL Lac objects, in which the peak of the synchrotron bump is located in the UV-X-ray bands and the high energy peak around $100 \mathrm{GeV}$ (these sources are often called high frequency peaked BL Lacs, HBLs). PG $1553+113$ is a BL Lac discovered by Green et al. (1986). The large $\mathrm{X}$-ray to radio flux ratio makes this source a typical HBL. Indeed, its synchrotron peak is located between the UV and X-ray bands. Its optical spectrum is featureless, preventing the direct determination of the redshift. Indirect methods based on the non detection of the characteristic lines and of the host galaxy provide lower limits, ranging from 0.09 to 0.78 (Sbarufatti et al. 2006; Sbarufatti, Treves, \& Falomo 2005). The most recent estimate, based on the Ly alpha forest method, gives a $z \sim 0.40-0.45$ (Danforth et al. 2010).

PG 1553+133 has been discovered as a VHE $\gamma$ ray emitter by H.E.S.S. (Aharonian et al. 2006a) and MAGIC (Albert et al. 2007a), with a flux of approximately $2 \%$ of that of the Crab Nebula above $200 \mathrm{GeV}$. The spectrum appears extremely soft (photon index $\Gamma \sim 4)$, as expected by the absorption of VHE photons through interaction with the Extragalactic Background Light (EBL) if the source is located at relatively large redshift (Stecker, de Jager, \& Salamon 1992). The absorption process, in fact, is a function of the energy of the photon and of the distance it has traveled. Spectra with indices $\Gamma \sim 4$ have been observed in blazars located at redshifts above 0.2 .

VHE $\gamma$ ray observations have been used as alternative method to constrain the distance of blazars. Aharonian et al. (2006b) proposed a way to set an upper limit on the distance of blazars based on the assumption that the VHE intrinsic spectrum, obtained by correcting the observed spectrum for the extragalactic background light absorption, cannot be harder than a fixed value given by theory. The technique, applied to PG $1553+113$, lead to an upper limit of $z<0.74$. Recently, Prandini et al. (2010) extended this method using the spectrum measured at lower energies as limiting slope for the original spectrum, obtaining $z<0.66$ for PG $1553+113$, at $2 \sigma$ level. Other approaches require the absence of a pile up at high energies. With this method, Mazin \& Goebel (2007) get $z<0.42$. Hence, in the case of PG $1553+113$, the upper limits obtained with these methods are in the range of the limits set by optical measurements. In this work we adopt the redshift $z=0.40$. Such a large redshift is also supported by the absence of significant points at energies above $700 \mathrm{GeV}$ in the spectrum of the source.

At MeV-GeV energies, PG 1553+113 was not detected by EGRET, but it is well visible by the Large Area Telescope (LAT) on-board Fermi, being detected with a significance above $10 \sigma$ already in the first three months of observations (Abdo et al. 2009a). Abdo et al. (2010b) show that the Fermi/LAT spectrum is surprisingly constant both in normalization and slope over $\sim 200$ days. Interestingly, a stability of the spectrum was also suggested by the H.E.S.S. and MAGIC observations, showing rather marginal variability during 2005 and 2006 observations. The stability of the $\mathrm{VHE} \gamma$ ray emission is in contrast to the behavior commonly observed in other $\mathrm{TeV}$ emitting BL Lacs, showing rather pronounced variations at all timescales.

After its discovery, PG $1553+113$ was regularly observed by MAGIC. In this paper, we present the analysis of the new data taken from 2007 to 2009, combined with previous observations. The differential and integral fluxes are analyzed, in comparison with partially simultaneous measurements at other wavelengths, and the stability of the spectrum over this long period is studied. Finally, we combine all the data available and model the SED with a one-zone Synchrotron Self Compton (SSC) model, constraining the main physical parameters that govern the VHE emission in the blazar jet.

\section{MAGIC OBSERVATIONS AND DATA ANALYSIS}

Since autumn 2009, MAGIC (Cortina et al. 2009) is a stereo system composed by two Imaging Atmospheric Cherenkov Telescopes (IACTs) located on La Palma, Canary Islands, Spain $\left(28.75^{\circ} \mathrm{N}, 17.89^{\circ} \mathrm{W}, 2240 \mathrm{~m}\right.$ asl). In this paper, we present only data collected before the stereo upgrade, with a single telescope, MAGIC I (Baixeras et al. 2004), hereafter called MAGIC. The parabolic-shaped reflector, with a total mirror area of 
$236 \mathrm{~m}^{2}$, allows MAGIC to collect the Cherenkov light and focus it onto a multi-pixel camera, composed of 577 photo-multipliers. MAGIC camera and trigger are designed to record data not only during dark nights, but also under moderate light conditions (i.e. moderate moon, twilight). Due to its comparatively low trigger energy threshold of $\sim 50 \mathrm{GeV}$, MAGIC is well suited to perform multiwavelength observations together with instruments operating in the $\mathrm{GeV}$ range.

TABLE 1

PG 1553+113 FINAL DATA SET

\begin{tabular}{cccccc}
\hline \hline Cycle & Date & $\begin{array}{c}\text { Eff. Time } \\
{[\mathrm{min}]}\end{array}$ & $\begin{array}{c}Z d \\
{\left[{ }^{\circ}\right]}\end{array}$ & $\begin{array}{c}\text { Rate } \\
{[\mathrm{Hz}]}\end{array}$ & $\begin{array}{c}\text { DC } \\
{[\mu \mathrm{A}]}\end{array}$ \\
\hline \hline \multirow{6}{*}{ III } & $23 / 03 / 2007$ & 58 & $19-29$ & 164 & dark night \\
& $19 / 04 / 2007$ & 32 & $22-28$ & 155 & dark night \\
& $20 / 04 / 2007$ & 150 & $17-29$ & 163 & dark night \\
& $21 / 04 / 2007$ & 115 & $17-24$ & 155 & dark night \\
& $22 / 04 / 2007$ & 101 & $17-23$ & 162 & dark night \\
& $23 / 04 / 2007$ & 143 & $17-27$ & 161 & dark night \\
& $24 / 04 / 2007$ & 92 & $17-23$ & 160 & dark night \\
\hline \multirow{6}{*}{ IV } & $17 / 03 / 2008$ & 58 & $17-19$ & 150 & dark night \\
& $18 / 03 / 2008$ & 26 & $18-19$ & 150 & dark night \\
& $01 / 04 / 2008$ & 43 & $20-26$ & 167 & dark night \\
& $05 / 04 / 2008$ & 109 & $17-31$ & 167 & dark night \\
& $13 / 04 / 2008$ & 97 & $17-22$ & 147 & dark night \\
& $29 / 04 / 2008$ & 44 & $27-36$ & 151 & dark night \\
& $03 / 05 / 2008$ & 24 & $26-31$ & 146 & dark night \\
& $04 / 05 / 2008$ & 40 & $28-36$ & 155 & dark night \\
$05 / 05 / 2008$ & 38 & $26-33$ & 150 & dark night \\
& $07 / 05 / 2008$ & 40 & $28-36$ & 153 & dark night \\
\hline \hline
\end{tabular}

${ }^{\text {a }} \mathrm{PG} 1553+113$ data set from 2007 to 2009 used in this study. From left to right: MAGIC Cycle of observation, first column, and corresponding dates in $\mathrm{dd} / \mathrm{mm} / \mathrm{yy}$, second column; effective time of observation in minutes and zenith angle range in degrees, third and fourth column. In the last two columns, the rate of the events after the image cleaning, in $\mathrm{Hz}$, and the mean $\mathrm{DC}$ current in the camera, in unit of $\mu \mathrm{A}$, are shown. The night is considered as dark night, if the DC current while observing an extragalactic object is less than indicatively $1.2 \mu \mathrm{A}$.

The total Field of View (FoV) of the MAGIC camera is $3.5^{\circ}$, and the effective collection area is of the order of $10^{5} \mathrm{~m}^{2}$ at $200 \mathrm{GeV}$ for a source close to zenith. The incident light pulses are converted into analog signals, transmitted via optical fibers and digitised by $2 \mathrm{GHz}$ fast analog to digital converters (FADCs).

PG 1553+113 was observed with the MAGIC telescope for nearly 19 hours in 2005 and 2006 (Albert et al. 2007a); it was also the subject of a multiwavelength campaign carried out in July 2006 with optical, X-ray and $\mathrm{TeV} \gamma$ ray telescopes (Albert et al. 2009). Here, we present the results of follow-up observations, performed for 14 hours in March-April 2007, for nearly 26 hours in March-May 2008, some of those simultaneously with other instruments (Aleksić et al. 2010), and for about 24 hours in March-July 2009, which were partly taken in moderate light conditions (moon light). Unfortunately, both 2008 and 2009 observations were severely affected
TABLE 2

PG $1553+113$ SIGNAL

\begin{tabular}{c|c|c|c|c|c}
\hline \hline Year & $\begin{array}{c}\text { Time } \\
{[\mathrm{h}]}\end{array}$ & $\begin{array}{c}\text { Opt. PSF } \\
{[\mathrm{mm}]}\end{array}$ & $\begin{array}{c}\text { Energy Th. } \\
{[\mathrm{GeV}]}\end{array}$ & Excesses & $\begin{array}{c}\text { Signif. } \\
{[\sigma]}\end{array}$ \\
\hline \hline 2007 & 11.5 & 13 & 80 & $1400 \pm 242$ & 5.8 \\
\hline 2008 & 8.7 & 13 & 150 & $542 \pm 69$ & 8.1 \\
\hline 2009 & 8.5 & 14.8 & 160 & $212 \pm 52$ & 4.2 \\
\hline \hline
\end{tabular}

${ }^{\text {a }}$ PG $1553+113$ signal study. From left to right: year of observation, effective time of good quality data used for the signal analysis, optical point spread function (PSF), energy threshold of the analysis, number of excess events observed and significance of the signal.

by bad weather (including calima, i.e. Saharan sanddust in the atmosphere) that limited the final data set and resulted in an increased energy threshold.

All data analyzed here were taken in the false-source tracking (wobble) mode (Fomin et al. 1994), in which the telescope pointing was alternated every 20 minutes between two sky positions at $0.4^{\circ}$ offset from the source. The zenith angle of 2007 observations varied from $17^{\circ}$ to $30^{\circ}$, in 2008 it extended up to $36^{\circ}$, while in 2009 it covered the range from $17^{\circ}$ to $35^{\circ}$.

The data were analyzed using the standard MAGIC analysis chain (Albert et al. 2008a; Aliu et al. 2009). Severe quality cuts based on event rate after night sky background suppression were applied to the sample; 28.7 hours of good quality data remained after these cuts, out of which 11.5 hours were taken in 2007, 8.7 hours in 2008 and 8.5 hours in 2009. More details about the final data set can be found in Table 1. For the signal study, a cut in the parameter size removed events with a total charge less than 80 photo-electrons (phe) in the 2007 data set, and 200 phe in 2008 and 2009 data sets. In the latter case, this cut reduces the effect of the moon light.

Finally, for the spectrum determination an additional cut in PMT DC current, namely above $2.5 \mu \mathrm{A}$, was applied to the 2009 sample, in order to reduce systematics due to the moon light (Britzger et al. 2009), resulting in 6.9 hours of good quality data. For the conclusive steps of the analysis, Monte Carlo (MC) simulations of $\gamma$-like events were used. Hadronic background suppression was achieved using the Random Forest (RF) method (Albert et al. 2008c), in which each event is assigned an additional parameter, the hadronness, which is related to the probability that the event is not $\gamma$-like. The RF method was also used in the energy estimation. The threshold of the analysis was estimated to be $80 \mathrm{GeV}$ in $2007,150 \mathrm{GeV}$ in 2008 and $160 \mathrm{GeV}$ in 2009 , as shown in Table 2.

Due to changes in the telescope performance, the sigma of the optical point-spread function (PSF) of 2007 and 2008 was measured to be $13.0 \mathrm{~mm}$, while in 2009 it was $14.9 \mathrm{~mm}$. To take all these differences into account, the data were analysed separately, using dedicated sets of simulated data.

\section{VHE $\gamma$ RAY RESULTS}

The 28.7 hours of good quality observations of PG 1553+113 carried out between 2007 and 2009 resulted in a signal of $8.8 \sigma$ of significance according to eq. 17 of $\mathrm{Li} \& \mathrm{Ma}(1983)$, obtained by combining the results from each year, listed in Table 2 The signal was extracted by analyzing the distribution of the parameter 
TABLE 3

PG 1553+113 MEASURED SPECTRA

\begin{tabular}{c|c|c|c|c}
\hline \hline Year & $\begin{array}{c}F>150 \mathrm{GeV} \\
{\left[\mathrm{cm}^{-2} \mathrm{~s}^{-1}\right]}\end{array}$ & $\begin{array}{c}F>150 \mathrm{GeV} \\
{[\text { Crab } \%]}\end{array}$ & $\begin{array}{c}\mathrm{f}_{0} \\
{\left[\mathrm{~cm}^{-2} \mathrm{~s}^{-1} \mathrm{TeV}^{-1}\right]}\end{array}$ & $\Gamma$ \\
\hline \hline 2007 & $(1.40 \pm 0.38) \cdot 10^{-11}$ & $4 \%$ & $(1.1 \pm 0.3) \cdot 10^{-10}$ & $4.1 \pm 0.3$ \\
\hline 2008 & $(3.70 \pm 0.47) \cdot 10^{-11}$ & $11 \%$ & $(2.6 \pm 0.3) \cdot 10^{-10}$ & $4.3 \pm 0.4$ \\
\hline 2009 & $(1.63 \pm 0.45) \cdot 10^{-11}$ & $5 \%$ & $(1.3 \pm 0.2) \cdot 10^{-10}$ & $3.6 \pm 0.5$ \\
\hline \hline
\end{tabular}

Note. - Spectra of the individual years of observations of PG 1553+113. From left to right: Year of MAGIC observations; Effective time in hours; Integral flux above $150 \mathrm{GeV}$ in units of $\mathrm{cm}^{-2} \mathrm{~s}^{-1}$ and Crab Nebula \%, normalization factor $\mathrm{f}_{0}$ in units of $\mathrm{cm}^{-2} \mathrm{~s}^{-1} \mathrm{TeV}^{-1}$; in the last column, the $\Gamma$ index obtained by fitting the observed differential spectrum with a power law. The errors reported are statistical only. The systematic uncertainty is estimated to be $35 \%$ in the flux level and 0.2 in the power index.

alpha, related to the incoming direction of the primary cosmic ray inducing the atmospheric shower. More details on the signal extraction with the alpha technique can be found in Albert et al. 2008b. For the signal detection, no cut in energy was applied.

The significance of the signal was $5.8 \sigma$ in $2007,8.1 \sigma$ in 2008 and $4.2 \sigma$ in 2009. Due to a large difference in the energy thresholds and changes in the experimental conditions, the obtained fluxes cannot be compared directly. A detailed spectral analysis is necessary in order to study the source emission.

\subsection{Integral Flux}

In order to explore the $\mathrm{VHE} \gamma$ ray emission of PG 1553+113 from each year, we compared the integral flux above $150 \mathrm{GeV}$. This value is a safe compromise taking into account the different energy thresholds. The final samples and the results of the spectral analyses are shown in Table 3 .

The integral fluxes measured above $150 \mathrm{GeV}$ lie in the range of $4 \%$ to $11 \%$ of the Crab Nebula flux measured by MAGIC (Albert et al. 2008a): the highest flux level is recorded in 2008 ( 0.11 Crab unit:T), a factor between two to three larger compared to the one measured in 2007 (0.04 Crab units) and 2009 (0.05 Crab units). A constant fit to the data has a probability smaller than $0.001 \%$. Such changes in the flux level observed in PG $1553+113$ are quite moderate in comparison to other monitored $\mathrm{TeV}$ blazars. For example, in Mkn 421 a flux variations exceeding one order of magnitude have been observed (e.g. Fossati et al 2008).

A detailed study about possible flux level variations on short timescale was carried out with the limiting condition that the signal is not strong enough to allow for a detailed sampling on sub-day timescale. The upper panel of Figure 1 displays the light curve of PG 1553+113 measured from 2007 to 2009 by MAGIC with a variable binning. For comparison, the daily flux levels measured in 2005 and 2006 are shown, as extrapolated from the published data (Albert et al. 2007a), and rescaled according to the power laws that interpolate the differential fluxes. Furthermore, the 2006 mean integral flux above $150 \mathrm{GeV}$ taken during the multiwavelength campaign and reported in Albert et al. (2009) is shown. The former data have not been used for the integral flux study, due to very large uncertainties related to the extrapolation procedure. We set 2-days, daily and monthly binning

1 The Crab unit used in this work is an arbitrary unit obtained by dividing the integral energy flux measured above a certain threshold by the Crab Nebula flux measured above the same threshold by MAGIC (Albert et al. 2008a). for the 2007, 2008, and 2009 data sets respectively, according to the significance of the signal. The 2008 data are consistent with the hypothesis of constant flux with a probability of $50 \%$ (Figure 2, lower panel).

In 2007, the observed time series is consistent with constant flux (93\% of probability). Nothing similar can be concluded for the 2009 observations since the significance of the signal is too low.

In general, the high energy threshold of the analysis together with the weakness of the PG $1553+113$ signal and its very steep spectrum make any variability study at short timescale difficult and might have hidden the detection of an increased activity on very short timescale.

\subsection{Differential Flux}

The differential spectra observed from PG $1553+113$ by MAGIC each year from 2007 to 2009 are shown in the left plot of Figure 3 .

As for other blazars, each spectrum can be well fitted with a power law function of the form

$$
\frac{d F}{d E}=f_{0} *\left(\frac{E}{200 \mathrm{GeV}}\right)^{-\Gamma}
$$

where $f_{0}$ is the flux at $200 \mathrm{GeV}$ and $\Gamma$ is the power law index. The resulting indices are listed in the last column of Table 3. The systematic uncertainty is estimated to be $35 \%$ in the flux level and 0.2 in the power index (Albert et al. 2008a), and is the sum of many contributions, mainly related to the use of MC simulations instead of test beams. Thanks to the low energy threshold of the analysis of 2007 data, the corresponding spectrum has a measured point below $100 \mathrm{GeV}$. In particular, the measured differential energy flux at $98 \mathrm{GeV}$ is $(2.7 \pm 0.3) \cdot 10^{-9} \mathrm{~cm}^{-2} \mathrm{~s}^{-1} \mathrm{TeV}^{-1}$, in agreement, within the errors, with the low energy point measured in 2005 and $2006,(4.1 \pm 1.2) \cdot 10^{-9} \mathrm{~cm}^{-2} \mathrm{~s}^{-1} \mathrm{TeV}^{-1}$ at $97 \mathrm{GeV}$.

The 2008 differential energy spectrum measured above $150 \mathrm{GeV}$ has a slope of $4.3 \pm 0.4$, while the slope of the spectrum determined with a partially simultaneous sample taken during a multiwavelength campaign with other instruments is $3.4 \pm 0.1$ between 70 to $350 \mathrm{GeV}$ (Aleksić et al. 2010). The different energy range characterizing the measurements fully accounts for this apparent disagreement: the spectral points measured in the range $150-350 \mathrm{GeV}$ are, in fact, in very good agreement.

Finally, the 2009 differential spectrum is barely determined due to the limited signal. Except for the latter sample, whose significance is rather low and corresponding errors noticeably large, the power law indices describing the spectra are compatible. This indicates that the shape of the emitted spectrum does not change, even if 


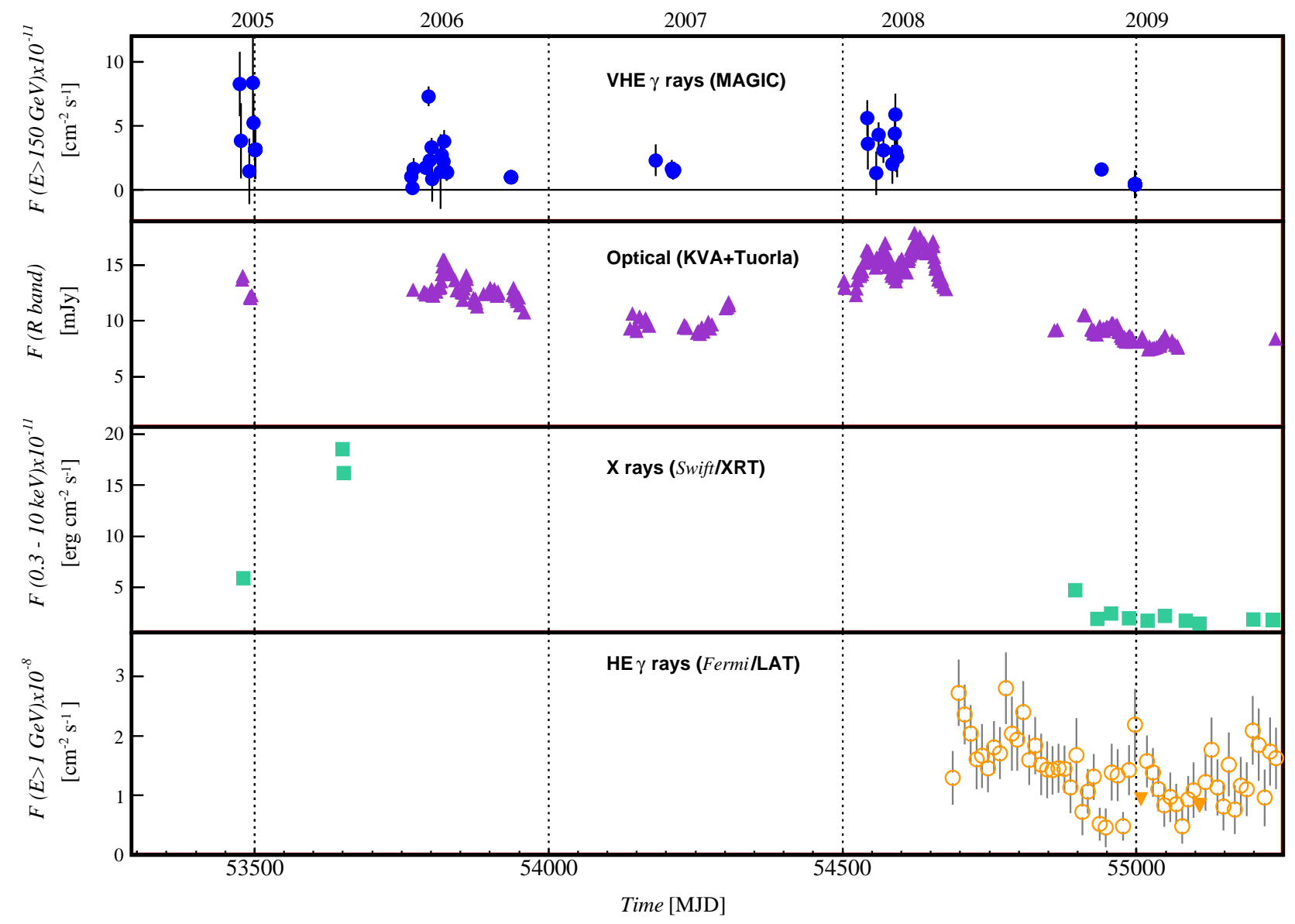

FIG. 1.- Multiwavelength light curve of PG 1553+113 from 2005 to late 2009. From upper to lower panel: VHE $\gamma$ rays above $150 \mathrm{GeV}$ measured by MAGIC (filled circles), optical flux in the R-band (triangles), X rays $0.3-10 \mathrm{keV}$ flux (squares) and $\gamma$ rays above $1 \mathrm{GeV}$ (open circles). The error bars reported have $1 \sigma$ significance. Downward triangles, in lower panel, refer to 2 sigma upper limits on the source flux above $1 \mathrm{GeV}$.

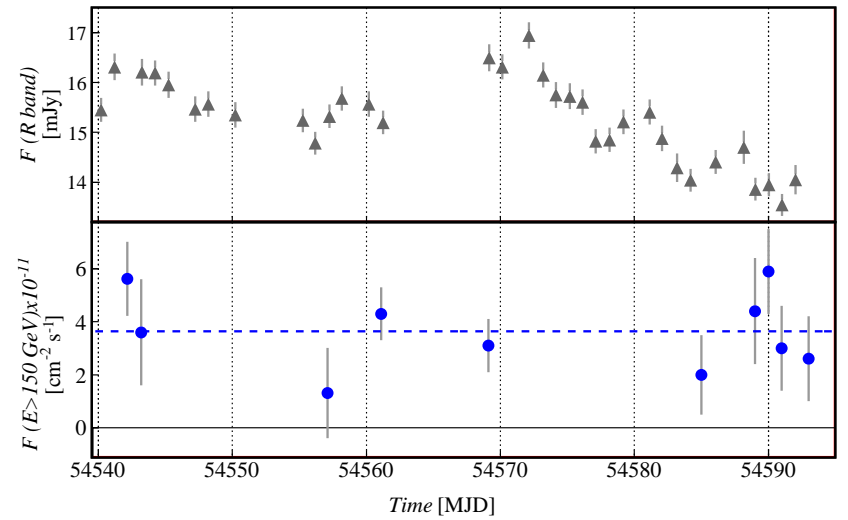

FIG. 2.- Zoom of Figure 1 around 2008 MAGIC observations (lower panel). In the upper panel the corresponding optical flux has been superimposed for comparison.

the total flux shows hints of (small amplitude) variability, as also noted for other BL Lacs such as 1ES 1218+304 (Acciari et al. 2010).

The right plot of Figure 3 shows the combined differential spectrum of PG $1553+113$ from 2007 to 2009, superimposed to the 2005-2006 spectrum measured by MAGIC $(\Gamma=4.21 \pm 0.25$, Albert et al. 2007a). The gray band represents the systematic effect on the combined spectrum resulting from the use of different methods to correct for the effects due to the finite energy resolution (procedure called unfolding, Albert et al. (2007b)). The good agreement among these mean determinations suggests that despite the (small) variability seen on yearly scale, the mean flux emitted by this source is stable. A power law fit gives the values $\Gamma=4.27 \pm 0.14$ for the index and $\mathrm{f}_{0}=(1.61 \pm 0.14) \cdot 10^{-10} \mathrm{~s}^{-1} \mathrm{~cm}^{-2} \mathrm{TeV}^{-1}$ for the normalization factor, with a $\chi^{2} /$ dof $=5.7 / 9$ and corresponding probability of $77 \%$. The integral flux above $150 \mathrm{GeV}$ is at the level of $8 \%$ of the Crab Nebula flux.

VHE photons from cosmological distances are absorbed in the interaction with the EBL. Taking into account the EBL absorption assuming the background model proposed in Dominguez et al. (2011) and a redshift $z=0.40$, the intrinsic spectrum is compatible with a power law of index $3.09 \pm 0.20$, as drawn in Figure 3 . If we assume $z=0.45$, the corresponding spectrum is compatible with a power law of index $2.91 \pm 0.21$.

\section{PG $1553+113$ AS SEEN AT OTHER WAVELENGTHS}

Figure 1 displays the light curve of PG $1553+113$ in different wavelengths. The MAGIC data shown cover five cycles of $\mathrm{TeV}$ observations at energies above $150 \mathrm{GeV}$. The time bins used are variable, as described in the previous section.

The simultaneous optical R-band data are outlined in the second panel. These data are collected on a nightly basis by the Tuorla Observatory Blazar Monitoring Pro- 

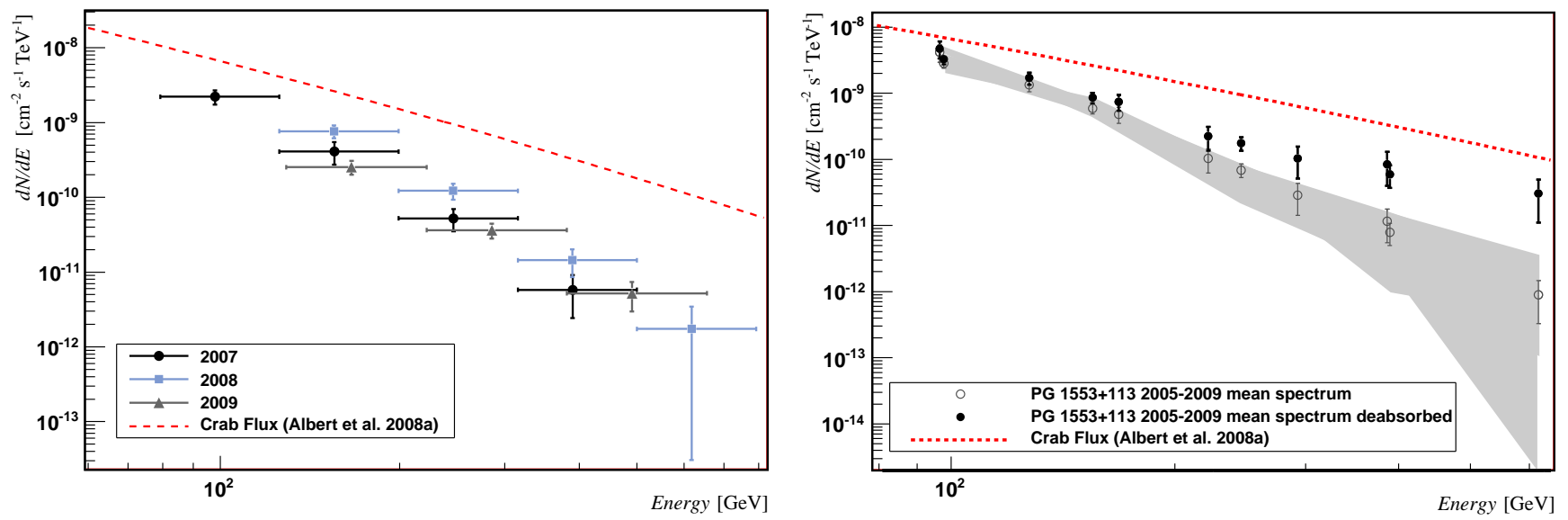

Fig. 3.- Differential energy spectra from PG 1553+113. Left Figure: comparison between 2007, 2008 and 2009 spectra. Right Figure: superimposition of 2005-2006 spectrum, from Albert et al. (2007a), to 2007-2009 mean spectrum and corresponding deabsorption for $z=0.4$ using the EBL model of Dominguez et al. (2011). In both figures, the fit of the Crab Nebula spectrum measured by MAGIC (Albert et al. 2008a) is superimposed for comparison.

gram 2 (Takalo et al. 2007) using the KVA $35 \mathrm{~cm}$ telescope at La Palma and the Tuorla 1 meter telescope in Finland.

For the third panel in Fig 1 we used the 14 Swift pointed observations (Gehrels et al. 2004) of PG 1553+113 performed from 2005-04-20 to 2010-02-05. We summed the data collected on 5 and 7 July 2009 in order to have enough statistics to obtain a good spectral fit. The XRT data were processed with standard procedures (xrtpipeline v0.12.6), filtering, and screening criteria by using the Heasoft package (v6.11). We consider the data collected in photon counting mode, and thus only XRT event grades 0-12 were selected.

Source events were extracted from a circular region with a radius of 20 pixels ( 1 pixel $\sim 2.36$ "), while background events were extracted from a circular region with radius of 50 pixels away from the source region. Some observations showed an average count rate of $>0.5$ counts $\mathrm{s}^{-1}$, thus pile-up correction was required. In that case we extracted the source events from an annular region with an inner radius from 2 to 7 pixels (depending on the source count rate and estimated by means of the PSF fitting technique, see Moretti et al. 2005) and an outer radius of 30 pixels. We extracted background events within an annular region centered on the source with radii 70 and 120 pixels. Ancillary response files were generated with xrtmkarf, and account for different extraction regions, vignetting and PSF corrections. We used the last spectral redistribution matrices in the Calibration database maintained by HEASARC. All spectra were rebinned with a minimum of 20 counts per energy bin to allow $\chi^{2}$ fitting within XSPEC (v12.7.0; Arnaud 1996). We fit the spectrum with an absorbed (model tbabs in Xspec) log parabola law (see e.g. Tramacere et al. 2007), with a neutral hydrogen column fixed to its Galactic value $\left(3.65 \times 10^{20} \mathrm{~cm}^{-2}\right.$; Kalberla et al. 2005). The observed 0.3-10 keV fluxes obtained by Swift/XRT in the different observations are reported in the third panel of Fig. 1.

In the lower panel, the Fermi/LAT light curve of PG 1553+113, computed in 10-day bins, is displayed.

\footnotetext{
${ }^{2}$ More information at http://users.utu.fi/kani/1m/
}

Fermi data presented in this paper are restricted to the $1 \mathrm{GeV}-100 \mathrm{GeV}$ energy range and were collected from MJD 54682 (2008 August 4) to MJD 55200 (2010 January 4) in survey mode.

An unbinned analysis was performed to produce the light curve with the standard analysis tool gtlike, included in the Science Tools software package (version v09r21p00). P6_V11 DIFFUSE Instrument Response Functions (IRFs) were used, which are a refinement to previous analyses reflecting improved understanding of the point spread function and effective area (Abdo et al. 2011, in preparation). For this analysis, only photons belonging to the Diffuse class and located in a circular Region Of Interest (ROI) of $10^{\circ}$ radius, centered at the position of PG $1553+113$, were selected. In addition, we excluded photons arriving from zenith angles $>105^{\circ}$ to limit contamination from Earth limb $\gamma$ rays, and photons with rocking angle $>52^{\circ}$ to avoid time intervals during which Earth entered the LAT FoV.

A separate analysis of the high energy emission in each time bin was performed. All point sources in the 1FGL within 15 degrees of PG $1553+113$, including the source of interest itself, were considered in the analysis. Those within the 10 degree radius ROI were fitted with a power law with spectral indices frozen to the values obtained from the likelihood analysis of the full data set, while those beyond 10 degree radius ROI had their values frozen to those found in 1FGL.

Upper limits at $2 \sigma$ confidence level (downward triangles in Figures 1 and 4) were computed for time bins with Test Statistics (TS) $3<4$, and were handled as in the first Fermi/LAT catalog paper. The estimated systematic uncertainty on the flux is $10 \%$ at $100 \mathrm{MeV}, 5 \%$ at $500 \mathrm{MeV}$, and $20 \%$ at $10 \mathrm{GeV}$.

As already noted, during the five years of monitoring the source generally showed a marginal activity in the VHE $\gamma$ ray band. The same behavior is followed by the optical flux, whose variations are limited within a factor of four, with a maximum flux reached in 2008 and a minimum value in 2009. A low emission in 2009 is also registered at all the other wavelengths, Figure 4.

3 TS is 2 times the difference of the $\log$ (likelihood) with and without the source (Mattox et al. 1996). 


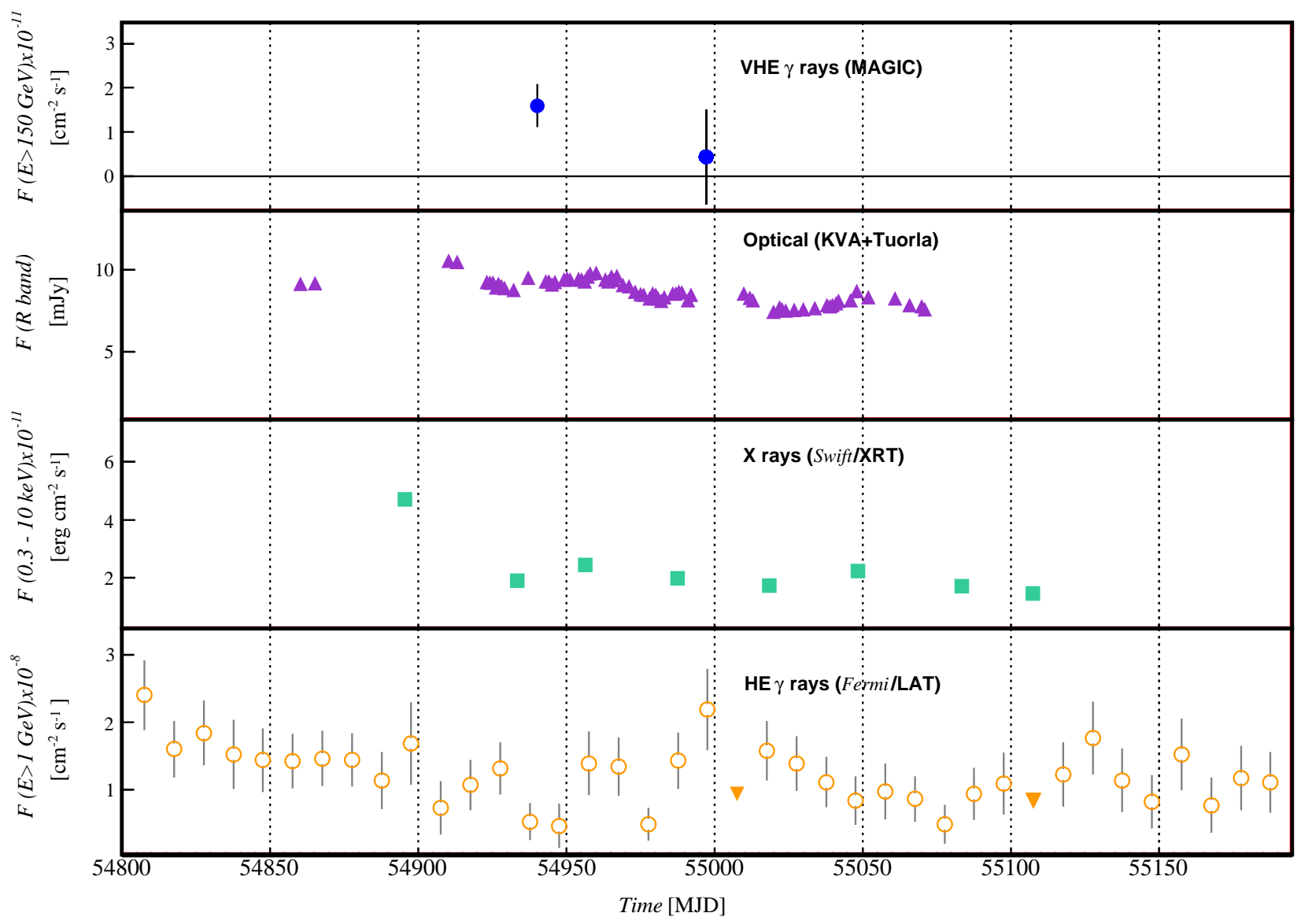

FIG. 4.- Zoom of Figure1 1 around the Cycle V observations carried out in 2009. From upper to lower panel: VHE $\gamma$ rays above $150 \mathrm{GeV}$ measured by MAGIC (filled circles), optical flux in the R-band (triangles), X rays $0.3-10 \mathrm{keV}$ flux (squares) and soft $\gamma$ rays above $1 \mathrm{GeV}$ (open circles). Downward triangles, in lower panel, refer to 2 sigma upper limits on the source flux above $1 \mathrm{GeV}$.

suggesting that the source entered in a low activity state during that year, with a minimum reached few days after MAGIC observations.

Figure 5 shows the result of a correlation study between optical and $\mathrm{TeV}$ simultaneous observations. The VHE $\gamma$ ray flux above $150 \mathrm{GeV}$ is plotted as a function of the optical flux. In order to increase statistics, we used for $2007 / 8 / 9$ samples the daily light curve values; however, since the optical measurements have a different time coverage, in some cases we derived the mean VHE flux from two or more consecutive days. 2005 and 2006 data, from Albert et al. (2007a), were rejected from this study, due to the large uncertainty on the extrapolated flux in the VHE band. The mean flux value from 2006 multiwavelength campaign, reported in Albert et al. (2009), is included. A linear relation among the two components has a $74 \%$ probability, which suggests a correlation between these two extreme energy bands. This result is in good agreement with the SSC model, which predicts a correlation between the synchrotron and the IC emission, related to the same electron population. Due to the poor simultaneity of VHE data with the other wavelengths, the same study has not been performed in X-rays and soft $\gamma$ rays.

The X-ray light curve shows a pronounced variability, in contrast to optical and very high energy bands. The X-ray flux spans an interval of about one order of magnitude (with maximum in 2005 and minimum in 2009),

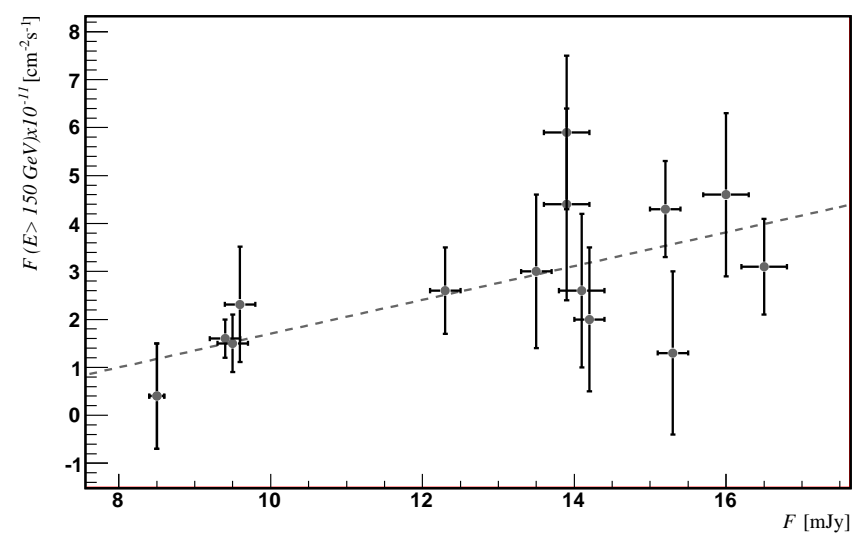

FIG. 5.- Correlation study between PG $1553+113$ optical Rband flux and VHE $\gamma$ ray integral flux above $150 \mathrm{GeV}$ observed from 2006 to 2009 .

larger than that observed in the $\mathrm{TeV}$, optical and $\mathrm{GeV}$ bands. The different variability displayed by the synchrotron (X-ray) and inverse Compton (GeV-TeV) components seems to be somewhat in contrast with the typical behavior observed in TeV BL Lacs, showing, in general, a coordinated variability (e.g. Fossati et al 2008)4. However, the sparse sampling of the observations and

${ }^{4}$ Rare exceptions to this rule are the so called "orphan" $\mathrm{TeV}$ flares, e.g. Krawczynski et al. (2004) 
the lack of a truly simultaneous monitoring prevents any strong conclusion. In particular, no optical nor gammaray data are available during the period of the maximum $\mathrm{X}$-ray flux in 2005. Coordinated multifrequency monitoring is necessary to further investigate this important issue.

In a dedicated paper (Abdo et al. 2010b), the Fermi/LAT collaboration reported the analysis of the first year of PG $1553+113$ data. They found that during the monitoring period the emission above $200 \mathrm{MeV}$ is almost steady. This is in contrast with the behavior of the source at higher energies $(>1 \mathrm{GeV})$. In our analysis of 2008 and 2009 LAT data (drawn in lower panel of Figure 11), in fact, a steady emission above $1 \mathrm{GeV}$ has a probability smaller than $0.1 \%$ and is ruled out. The lowest flux, observed in April 2009 , has a value $(0.5 \pm 0.3) \cdot 10^{-8} \mathrm{~cm}^{-2} \mathrm{~s}^{-1}$, while the highest flux, detected in August 2008, has a value $(2.8 \pm 0.6) \cdot 10^{-8} \mathrm{~cm}^{-2} \mathrm{~s}^{-1}$, more than 5 times higher. In our case, we are looking only at the upper edge of LAT band, probably close to the IC peak, while the integral flux above $200 \mathrm{MeV}$ reported by Abdo et al. (2010b) is dominated by lower energies, due to larger statistics. Therefore, we conclude that while at low energies (200 MeV-1 GeV) the IC continuum shows only marginal variability, this is not the case in the vicinity of the peak (some GeVs). In fact, small variability at $\mathrm{GeV}$ energies is a common feature of HBLs (Abdo et al. 2010a).

\section{MODELING THE SED}

In Figure 6. we assembled the SED of PG 1553+113 using historical data and the MAGIC spectra described above. Open black squares displaying radio-optical data are from NED5. In the optical band, we also show (red diamonds) the KVA minimum and maximum flux measured in the period covered by MAGIC 20052009 observations together with optical-UV fluxes from Swift/UVOT (filled black triangles, from Tavecchio et al. 2010). For the X-ray data, two Swift/XRT spectra taken in 2005 (high flux state, red crosses, and intermediate state, black asterisks, from Tavecchio et al. 2010) are given, and a Suzaku spectrum taken in 2006 (continuous red line, from Reimer et al. 2008). In addition, the average $15-150 \mathrm{keV}$ flux measured by Swift/BAT during the first 54 months of survey (Cusumano et al. 2010) is shown (black star), and the average $R X T E / \mathrm{ASM}$ flux between March 1 and May 31, 2008 (small black square), from quick-look results provided by the $R X T E / \mathrm{ASM}$ team 6 .

The green triangles correspond to the LAT spectrum averaged over $\sim 200$ days (2008 August-2009 February) from Abdo et al. (2010b). As discussed in that paper, the flux above $200 \mathrm{MeV}$ is rather stable, showing very small variability over the entire period of LAT observations. It is likely that the variability observed at the highest energies is not important in determining the averaged spectrum due to the limited statistics.

For MAGIC, we report the 2005-2006 and 2007-2009 observed spectra (filled circles) and the same spectra corrected for the absorption by the EBL using the model of Dominguez et al. (2011) (red open circles).

\footnotetext{
${ }^{5}$ http://nedwww.ipac.caltech.edu/

${ }^{6}$ http://xte.mit.edu/asmlc/
}

We model the SED with the one-zone SSC model fully described in Maraschi \& Tavecchio (2003). The emission zone is supposed to be spherical with radius $R$, in motion with bulk Lorentz factor $\Gamma$ at an angle $\theta$ with respect to the line of sight. Special relativistic effects are described by the relativistic Doppler factor, $\delta=[\Gamma(1-\beta \cos \theta)]^{-1}$. The energy distribution of the relativistic emitting electrons is described by a smoothed broken power law function, with limits $\gamma_{\min }$ and $\gamma_{\max }$ and break at $\gamma_{\mathrm{b}}$. To calculate the SSC emission, we use the full Klein-Nishina cross section.

Given the large variations of the X-ray synchrotron flux, we decided to use the average level of the synchrotron bump as measured by XRT, including also ASM and BAT fluxes to constrain the model. The corresponding input parameters are listed in Table 4. We also report the derived powers carried by the different components, relativistic electrons, $P_{e}$, magnetic field, $P_{B}$, and protons, $P_{p}$, (assuming a composition of one cold proton per relativistic electron) and the total radiative luminosity $L_{\mathrm{r}} \simeq L_{\mathrm{obs}} / \delta^{2}$.

In order to investigate the role of different parameters in the model, we have explored their variation as a function of the intensity of the synchrotron peak. To do so, we have modeled the SED considering the two extreme states of the synchrotron peak described above, respectively. For the SSC peak, instead, we have fixed the VHE data. The two curves representing the models are superimposed in light gray to Figure 6. The parameters obtained, listed in the last two columns of Table 4, are quite similar to the ones obtained when considering the average level of the synchrotron bump, except for the two variables $B$ and $K$, the magnetic field and the electron density. Indeed, these two parameters regulate the relative importance of synchrotron and SSC components. The state characterized by a low synchrotron emission has larger $B$ and smaller $K$ values with respect to the mean state modeled above. Conversely, the high synchrotron emission state has smaller values of $\mathrm{B}$ and a larger $\mathrm{K}$.

Finally, a comparison with the SED model obtained in the multiwavelength campaign reported in Aleksić et al. (2010), reveals that the parameters used for building the two models are quite similar. The major differences are the value of the Doppler factor, which in our model is relatively higher $(\delta=35)$ than in the previous one $(\delta=23)$, and that of the magnetic field $(0.5 \mathrm{G}$ instead of $0.7 \mathrm{G})$. This difference is mainly due to the higher SSC peak frequency that we find in our data, better defined by the combined LAT and MAGIC spectra.

The derived value of the total jet power, $P_{\text {jet }}=$ $P_{\mathrm{e}}+P_{B}+P_{\mathrm{p}}=4 \times 10^{44} \mathrm{erg} / \mathrm{s}$, is consistent with the typical values inferred modelling similar sources (e.g. Ghisellini et al. 2011). We use a relatively large minimum electron Lorentz factor $\gamma_{\min } \sim 10^{3}$ in order to reproduce the hard $\mathrm{MeV}-\mathrm{GeV}$ continuum tracked by LAT (photon index $\Gamma=1.68 \pm 0.03$ ). The high value of $\gamma_{\min }$ implies that, as commonly derived in TeV BL Lacs, the relativistic electrons (and the magnetic field, almost in equipartition) carry more power than the cold proton component. Another characteristic that PG $1553+113$ shares with the other TeV BL Lacs is that the total luminosity $L_{\mathrm{r}}$ is larger than the 
TABLE 4

INPUT MODEL PARAMETERS FOR THE MODELS SHOWN IN Fig. 6

\begin{tabular}{lcccc}
\hline \hline Parameter & & Value $_{\text {mean }}$ & Value $_{\max }$ & Value $_{\text {min }}$ \\
\hline \hline$\gamma_{\min }$ & {$\left[10^{3}\right]$} & 2.5 & 1 & 5 \\
$\gamma_{\mathrm{b}}$ & {$\left[10^{4}\right]$} & 3.2 & 3 & 1.3 \\
$\gamma_{\max }$ & {$\left[10^{5}\right]$} & 2.2 & 5.2 & 4.1 \\
$n_{1}$ & & 2.0 & 2.0 & 2.0 \\
$n_{2}$ & & 4.0 & 3.75 & 3.55 \\
$B$ & {$[\mathrm{G}]$} & 0.5 & 0.8 & 0.2 \\
$K$ & {$\left[10^{3} \mathrm{~cm}^{-3}\right]$} & 5.35 & 3.8 & 25 \\
$R$ & {$\left[10^{16} \mathrm{~cm}\right]$} & 1 & 1 & 1 \\
$\delta$ & & 35 & 35 & 35 \\
$P_{\mathrm{e}}$ & {$\left[10^{44} \mathrm{erg} / \mathrm{s}\right]$} & 2.2 & & \\
$P_{B}$ & {$\left[10^{44} \mathrm{erg} / \mathrm{s}\right]$} & 1.5 & & \\
$P_{\mathrm{p}}$ & {$\left[10^{44} \mathrm{erg} / \mathrm{s}\right]$} & 0.34 & & \\
$L_{\mathrm{r}}$ & {$\left[10^{44} \mathrm{erg} / \mathrm{s}\right]$} & 6.3 & & \\
\hline \hline
\end{tabular}

${ }^{a}$ We list for the three different models plotted in Fig. 6 the minimum, break and maximum Lorentz factors and the low and high energy slope of the electron energy distribution, the magnetic field intensity, the electron density, the radius of the emitting region and its Doppler factor. For the average model we also give the derived power carried by electrons, magnetic field, protons (assuming one cold proton per emitting relativistic electron) and the total radiative luminosity.

power supplied by electrons, magnetic field and protons. As discussed in Celotti \& Ghisellini (2008), this implies that either only a small fraction of leptons is accelerated at relativistic energies (leaving a reservoir of cold pairs and/or protons) or that the jet is dissipating a large fraction of its power as radiation, eventually leading to the deceleration of the flow, as in fact observed at VLBI scales (Piner et al. 2010) and envisaged in the models of structured jets Georganopoulos \& Kazanas 2003; Ghisellini et al. 2005).

\section{CONCLUSIONS}

In this paper, we presented the analysis of three years of VHE $\gamma$ ray data of PG $1553+113$ collected by MAGIC from 2007 to 2009 . The data set was divided into individual years, and a significant signal was found in every sample, confirming PG $1553+113$ as a stable presence in the VHE sky. The overall flux above $150 \mathrm{GeV}$ from 2007 to 2009 shows only a modest variability on yearly time-scale, within a factor 3 , corresponding to a variation between $4 \%$ to $11 \%$ of the Crab Nebula flux. No clear variability on smaller time scales is evident in the sample.

For the spectral analysis, the data set was combined with previous observations carried out by MAGIC during the first two Cycles of operations, from 2005 to 2006, for a total of five years of monitoring. This sample was excluded from the temporal study due to very large systematics related to the flux extrapolation procedure. Despite the hints of variability on the flux level, the differential flux from each year is in very good agreement with a power law of constant index $4.27 \pm 0.14$. This behavior has been already observed in other blazars, such as the HBL 1ES 1218+304 (Acciari et al. 2010).

PG 1553+113 was also monitored in optical, X-ray and soft $\gamma$ ray frequencies, but only the former data could be used for correlation studies thanks to the large timing coverage. Interestingly, a hint of correlation with probability of $74 \%$ was found between MAGIC and R-band optical flux levels, which in turn shows only a modest variability within a factor 4 . A clear variability is seen in the $\mathrm{X}$-rays and $\gamma$ rays above $1 \mathrm{GeV}$. The latter outcome, exploring the energies close to the IC peak, is only apparently in contradiction with previous results stating a quite stable spectrum for this source in the soft $(>200 \mathrm{MeV}) \gamma$-ray band (Abdo et al. 2010b). The different energy thresholds used in the two studies can, in fact, explain very well the discrepancy, as discussed in the paper.

Finally, for the study of the spectral energy distribution, the mean differential spectrum measured by MAGIC was combined with historical data at other wavelengths. Due to the large variations observed in X-rays and characterizing the synchrotron peak, we decided to use for the SED modeling the high energy bump, and the average level of the low energy bump. A more precise model requires coupling the VHE $\gamma$ ray part of the spectrum with simultaneous coverage of the synchrotron peak, in particular at optical-X-ray energies. An interesting feature of PG $1553+113$ is the narrowness of the SSC peak derived from the LAT and MAGIC spectra, implying a relatively large value of the minimum Lorentz factor of the emitting electrons, $2.5 \times 10^{3}$. This is also required by other HBLs with hard $\mathrm{GeV}$ spectra (e.g. Tavecchio et al. 2010).

The MAGIC stereo system, with its increased sensitivity and low energy threshold, is the suitable instrument to further investigate eventual daily scale $\mathrm{TeV}$ variability, as well as to provide a good differential spectrum determination below $100 \mathrm{GeV}$.

\section{ACKNOWLEDGEMENTS}

We would like to thank the Instituto de Astrofísica de Canarias for the excellent working conditions at the Observatorio del Roque de los Muchachos in La Palma. The support of the German BMBF and MPG, the Italian INFN, the Swiss National Fund SNF, and the Spanish MICINN is gratefully acknowledged. This work was also supported by the Marie Curie program, by the CPAN CSD2007-00042 and MultiDark CSD2009-00064 projects of the Spanish Consolider-Ingenio 2010 programme, by grant DO02-353 of the Bulgaria n NSF, by grant 127740 of the Academy of Finland, by the YIP of the Helmholtz Gemeinschaft, by the DFG Cluster of Excellence "Origin and Structure of the Universe", by the DFG Collaborative Research Centers SFB823/C4 and SFB876/C3, and by the Polish MNiSzW grant $745 / \mathrm{N}-$ HESS-MAGIC/2010/0.

The Fermi LAT Collaboration acknowledges generous ongoing support from a number of agencies and institutes that have supported both the development and the operation of the LAT as well as scientific data analysis. These include the National Aeronautics and Space Administration and the Department of Energy in the United States, the Commissariat à l'Energie Atomique and the Centre National de la Recherche Scientifique / Institut National de Physique Nucléaire et de Physique des Particules in France, the Agenzia Spaziale Italiana and the Istituto Nazionale di Fisica Nucleare in Italy, the Ministry of Education, Culture, Sports, Science and Technology (MEXT), High Energy Accelerator Research Organization (KEK) and Japan Aerospace Exploration Agency (JAXA) in Japan, and the K. A. Wallenberg Foundation, 


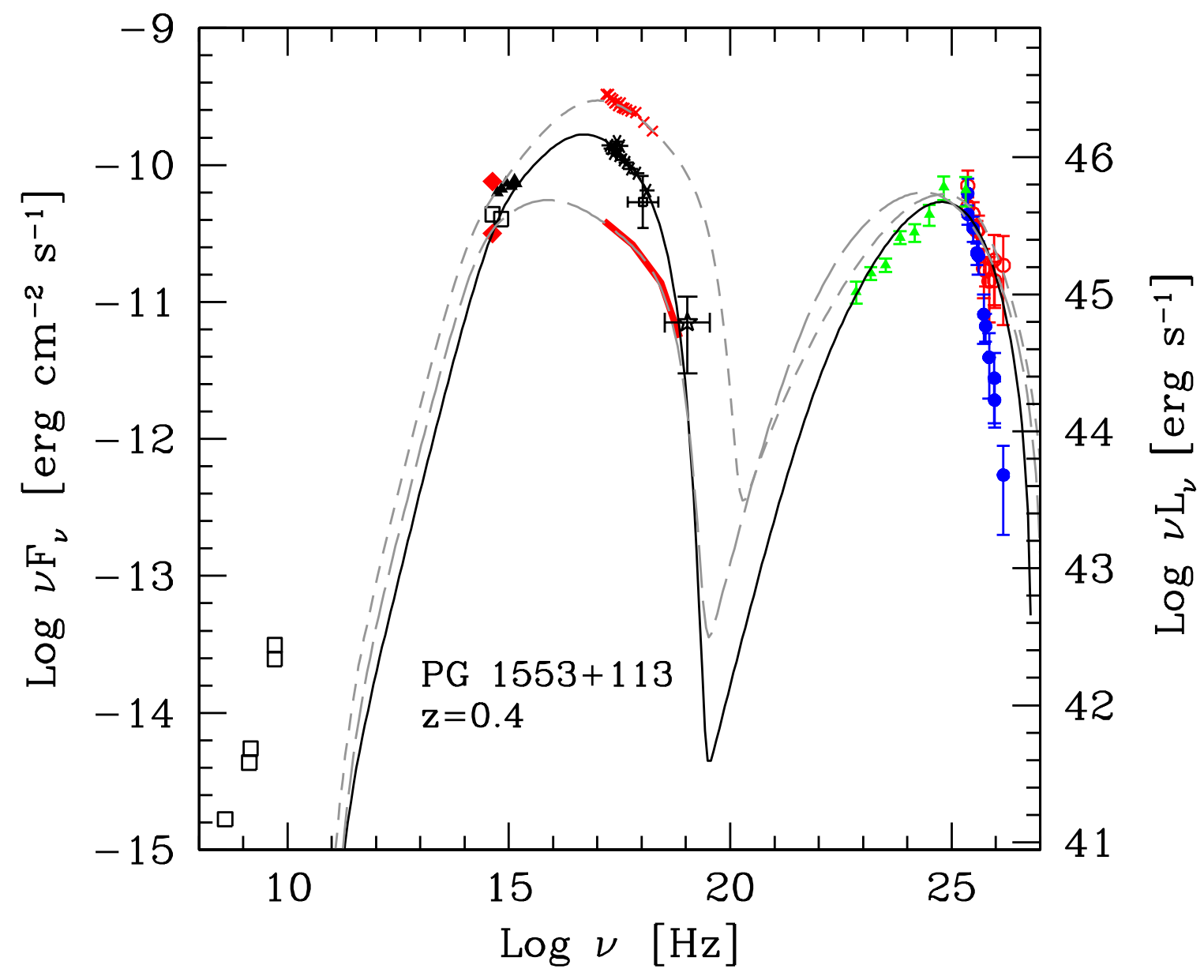

FIG. 6.- SED of PG 1553+113. Open black squares are radio-optical data from NED, red diamonds represent the KVA minimum and maximum flux measured in the period covered by MAGIC observations, together with optical-UV fluxes from Swift/UVOT (filled black triangles). For the X-ray data, two Swift/XRT spectra taken in 2005 (high flux state, red crosses, and intermediate state, black asterisks) are given, and a Suzaku spectrum taken in 2006 (continuous red line). The average 15-150 keV flux measured by Swift/BAT (black star) and the average RXTE/ASM flux between March 1 and May 31, 2008 (small black square), are also represented. The LAT spectrum averaged over $\sim 200$ days (2008 August-2009 February) is given (green filled triangles). For MAGIC, we display the 2005-2006 and 2007-2009 observed spectra (blue filled circles) and the same spectra corrected for the absorption by the EBL (red open circles). The average SED is modeled with a one-zone SSC model (continuous black line). Alternative SED are superimposed in light gray. Detailed references are addressed in the text.

the Swedish Research Council and the Swedish National Space Board in Sweden.

Additional support for science analysis during the operations phase is gratefully acknowledged from the Is- tituto Nazionale di Astrofisica in Italy and the Centre National d'Études Spatiales in France.

\section{REFERENCES}

Abdo, A. A., et al. 2010a, ApJ, 722, 520

Abdo, A. A., et al. 2010b, ApJ, 708, 1310

Abdo, A. A., et al. 2009, ApJ, 700, 597

Acciari, V. A., et al. 2010, ApJ, 709, L163

Aharonian, F., et al. 2006a, A\&A, 448, L19

Aharonian, F., et al. 2006b, Nature 440, 1018

Albert, J., et al. 2009, A\&A 493, 467

Albert, J., et al. 2008a, ApJ, 674, 1037

Albert, J., et. al. 2008b, ApJ, 685, L23
Albert, J., et al. 2008c, Nucl. Instr. Meth. A 588, 424

Albert, J., et al. 2007a, ApJ Letters, 654, L119

Albert, J., et al., 2007b, Nucl. Instr. Meth. A 583, 494

Aleksić, J., et al. 2010, A\&A 515, A76

Aliu, E., et al. 2009, ApJ, 30, 293

Arnaud, K. A. 1996, ASPC, 101, 17

Baixeras, C., et al. 2004, Nucl. Instrum. Meth. A 518, 188 
Britzger, D., Carmona, E., Majumdar, P., Blanch, O., Rico, J., Sitarek, J., \& Wagner, R. 2009, in Proc. 31st Int. Cosmic Ray Conf., preprint (arXiv:0907.0973)

Celotti, A., \& Ghisellini, G. 2008, MNRAS, 385, 283

Cortina, J., Goebel, F., \& Schweizer, T. 2009, preprint arXiv:0907.1211

Cusumano, G., et al. 2010, A\&A, 524, A64

Danforth,C. W., Keeney, A. B., Stocke, J. T., Michael Shull, J., \& Yao, J. 2010, ApJ, 720, 976

Dominguez et al., 2011, MNRAS, 410, 2556

Fomin, V. P., et al. 1994, ApJ, 2, 137

Fossati, G., et al. 2008, ApJ, 677, 906

Georganopoulos, M., \& Kazanas, D. 2003, ApJ, 594, L27

Gehrels, N., Chincarini, G., Giommi, P., et al. 2004, ApJ, 611, 1005

Ghisellini, G., et al. 2011, MNRAS, 414, 2674

Ghisellini, G., Tavecchio, F., \& Chiaberge, M. 2005, A\&A 432, 401

Green, R. F., Schmidt, M., \& Liebert, J. 1986, ApJS, 61, 305

Kalberla, P. M. W., Burton, W. B., Hartmann, D., et al. 2005, A\&, 440, 750
Krawczynski, H., et al. 2004, ApJ, 601, 151, 2004

Li, T. P., \& Ma, Y. Q. 1983, ApJ, 272, 317

Mannheim, K. 1993, A\&A, 269, 67

Mattox, J. R., et al., 1996, ApJ, 461, 396

Mazin, D., \& Goebel, F. 2007, ApJ Letters 655, L13

Maraschi, L., \& Tavecchio, F. 2003, ApJ, 593, 667-675

Moretti, A., Campana, S., Mineo, T., et al. 2005, SPIE, 5898, 360

Piner, B. G., Pant, N., \& Edwards, P. G. 2010

Prandini, E., Bonnoli, G., Maraschi, L., Mariotti, M., \&

Tavecchio, F. 2010, MNRAS 405, L76

Reimer, A., Costamante, L., Madejski, G., Reimer, O., \& Dorner, D. 2008, ApJ, 682, 775

Sbarufatti, B., Treves, A., Falomo, R., et al. 2006, ApJ, 132, 1

Sbarufatti, B., Treves, A., \& Falomo, R. 2005, ApJ, 635, 173

Stecker, F. W., de Jager, O. C. \& Salamon, M.H. 1992, ApJ 390, L49

Takalo, L. O., et al. 2007, ASP Series, 373, 249

Tavecchio, F., Ghisellini, G., Ghirlanda, G., Foschini, L., \&

Maraschi, L. 2010, MNRAS, 401, 1570

Tramacere, A., Massaro, F., Cavaliere, A. 2007, A\&A, 466, 521

Urry, C. M., \& Padovani, P. 1995, PASP, 107, 803 